\section{Fly Microdroplets Viewed Big: a Cryo-SEM Approach}

\author{
Stanislav N. Gorb \\ Max Planck Institute for Metals Research, Stuttgart, Germany \\ s.gorb@mf.mpg.de
}

\section{Introduction.}

There are numerous biological surfaces covered with secretory fluids, such as the adhesive pads of insects, sticky trichomes of plants, gustatory drops on plant leaves, lubricating coatings of vertebrate joints, etc. These fluids can be well visualised using light microscopy methods, however it is difficult to obtain detailed high magnification ultrastructural information on the morphology of these small-volume droplets. Conventional SEM preparation methods fail, because of the presence of vacuum and/or the instability of the subject fluid under the electron beam. Part of the problem can be resolved by the use of an Environmental SEM (ESEM) or an Atomic Force Microscopy (AFM), but the first method is restricted in its resolution and the second method has difficulty with resolving the complex geometry of the substrate and is rather slow for the analysis of these small, fast-evaporating droplets. Moreover, it is desirable in many applications to visualise the fluid behaviour at the contact interface between two surfaces, and this task cannot be resolved via the conventional application of either SEM or AFM methods. Herewith, we describe a Cryo-SEM approach successfully applied for visualisation of droplets of various fluids deposited on a substrate, or located at the interface between two surfaces.

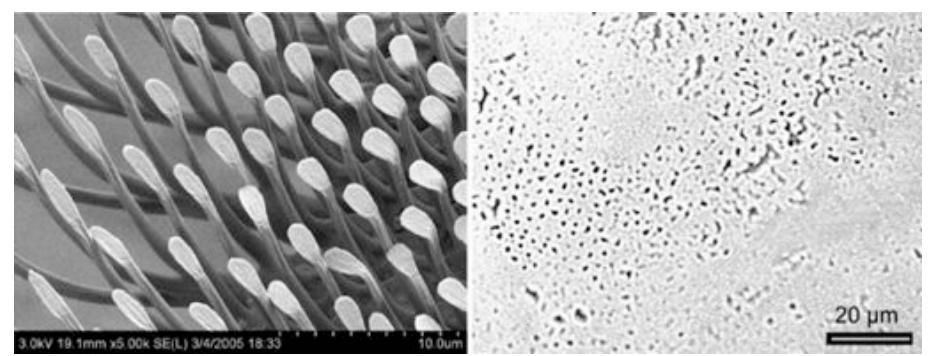

Figure 1. Adhesive organ of the fly. Left. Tenent hairs of the C. vicina fly viewed in the Cryo-SEM. Right. Fly footprints viewed in phase contrast mode in the light microscope, showing the limited ability of the optical microscope for detailed observation of small volumes of the fluid deposited on the surface.

\section{Fly footprints}

We have used an Hitachi S-4800 SEM equipped with a Gatan ALTO-2500 cryo preparation system. We started this project with a trial to visualise droplets of fly adhesive hairs (Figure $1 \mathrm{~A}$ ). These droplets are left behind by the fly after each step on a smooth wall or ceiling (Walker et al. 1985). The fluid is produced in the giant cells of its pulvillus (adhesive organ of the fly) and consists of a mixture of lipid-soluble and water-soluble components (Gorb 2001). The fluid is delivered in the contact area through a tiny channel running within each tenent hair bearing a little opening under the terminal plate close to the hair tip. Droplet size ranges from 1 to $3 \mu \mathrm{m}$. Light microscopy is a usefull method for visualise droplet patterns on a substrate (Figure $1 \mathrm{~B})$, but to observe details of the droplets, an electron microscopy approach is required.

In the first trial, we cut off a Calliphora vicina fly's tarsus and brought its adhesive organs into contact with glass coverslips by the use of fine forceps under a binocular microscope. The coverslip with the footprints was frozen in liquid nitrogen, transferred to the cryostage of the preparation chamber $\left(-140^{\circ} \mathrm{C}\right)$, and then sublimated at a temperature of $-90^{\circ} \mathrm{C}$ for $3 \mathrm{~min}$, to remove contamination by condensed ice crystals. The sample was coated in the frozen condition with gold- palladium ( $3 \mathrm{~nm}$ thickness) and observed at $\mathrm{t}$ $=-120^{\circ} \mathrm{C}$ and an SEM accelerating voltage of 1-3 kV. Even using the instrument's lower SE detector, which should be capable of excellent resolution of the very small differences in the substrate surface profile, we were hardly able to detect any droplets on the surface. Most of the trials to visualise fly footprints, clearly visible in the phase constrast mode of the light microscope, failed in this attempt.

\section{Method description}

In the second trial, we tried to increase the material contrast between the substrate and the footprints and endeavored to use the substrate profile to facilitate the observation of droplets at various angles. To fulfil these two requrements, we prepared drops (2-7 mm in diameter) of Spurr fluid metacrylate resin (Spurr 1969), usually used as an embeding medium for transmission electron microscopy, deposited on the flat, back-side of silicone embedding forms (Figure 2). (We determined that other kinds of resins, such as araldite, epon, and historesine can be used for this purpose as well.) After polymerization overnight at $+70^{\circ} \mathrm{C}$, the drops were sputter-coated with gold-palladium (6-10 $\mathrm{nm}$ thickness), as with coventional SEM preparation. Due to the surface tension of the fluid polymer, the droplet surface was exceptionally smooth and curved after polymerisation. These metal-coated resin drops were used as substrates for the fly footprint deposition. After the same footprint preparation procedures described above, the specimens were frozen, sublimated, and first observed uncoated in the Cryo-SEM. Since the organic footprints were located on the very smooth, sloped metal surface of the coated drops, the attendant high material contrast between the frozen fly footprint droplets and the surface allowed for easy observation of the frozen droplets. Droplets were viewed dark on a bright background (Figure 3).

Since the Gatan ALTO 2500 cryo preparation system allows backand-forth stepwise sample preparation, after the observation of uncoated droplets, we were able to bring the sample back to the preparation chamber, sputter-coat it with gold-palladium (thickness $=6 \mathrm{~nm}$ ) and observe it in the coated condition. This allowed us to localise footprints, however, the material contrast was reduced greatly due to the similar material coating of the footprint droplets and the substrate drops.

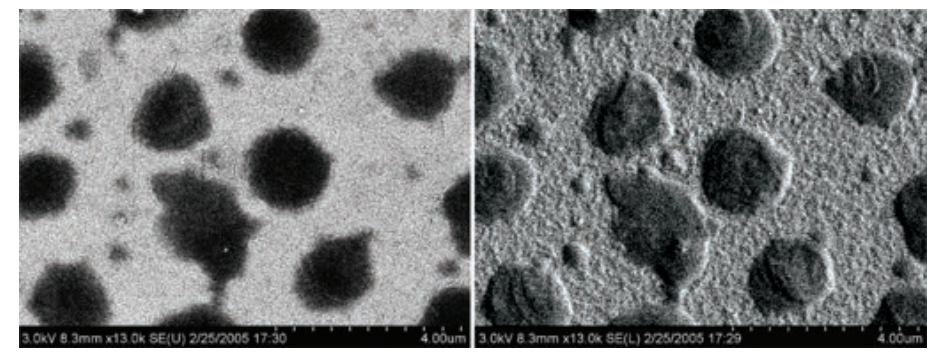

Figure 3. Uncoated frozen footprints of the fly C. vicina on the metallized resin-drop substrate. A. Upper SE detector. B. Lower SE detector. 


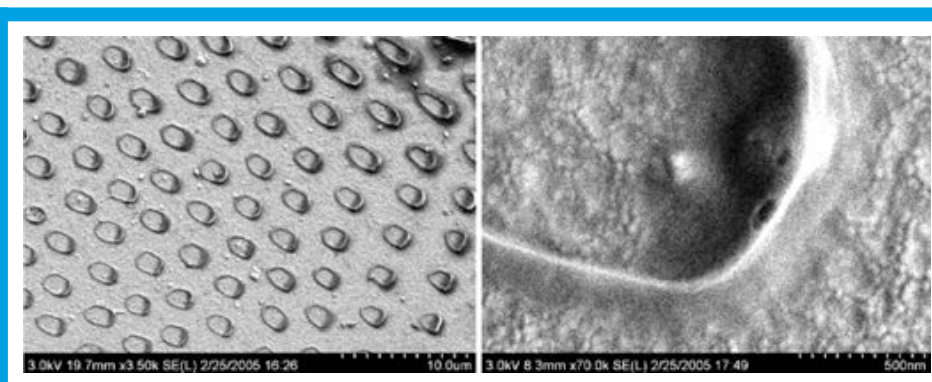

Figure 4. Uncoated footprints frozen while the adhesive hairs were in contact with the metallized substrate. After freezing, the adhesive organ was removed allowing for the observation fly footsteps in the fluid. The fluid menisci between each hair tip and the substrate can be seen. A. An array of prints corresonding to the pattern of tenent hairs of the sdhesive organ. B. Meniscus of the print of the single hair tip.

\section{Droplets at the interface}

Our most challenging task was the visualiation of droplets at the interface between hair tips and the substrate. For this purpose, freshly cut tarsi with adhesive hairs still in place were manually brought into contact with metallized Spurr drops and frozen in liquid nitrogen. In the preparation chamber, the tarsi were removed using a precooled metal blade normally applied for fracturing frozen samples. This approach allowed us to observe the adhesive fluid at the interface at the tips of tenent hairs (Figure 4). Figure 5 shows a colorized image of footprints and an inset showing the tenant hairs in contact with the substrate.

\section{Conclusion}

The methods described here have also been successfully applied to the visualisation of droplets of glycerin and water-glycerin mixtures as well as the adhesive fluids of plants. Additionally, the method allows a coarse estimation of the contact angle of the fluid on the metallized surface, which can be an important source of information for understanding the physico-chemical properties of the adhesive fluid. The

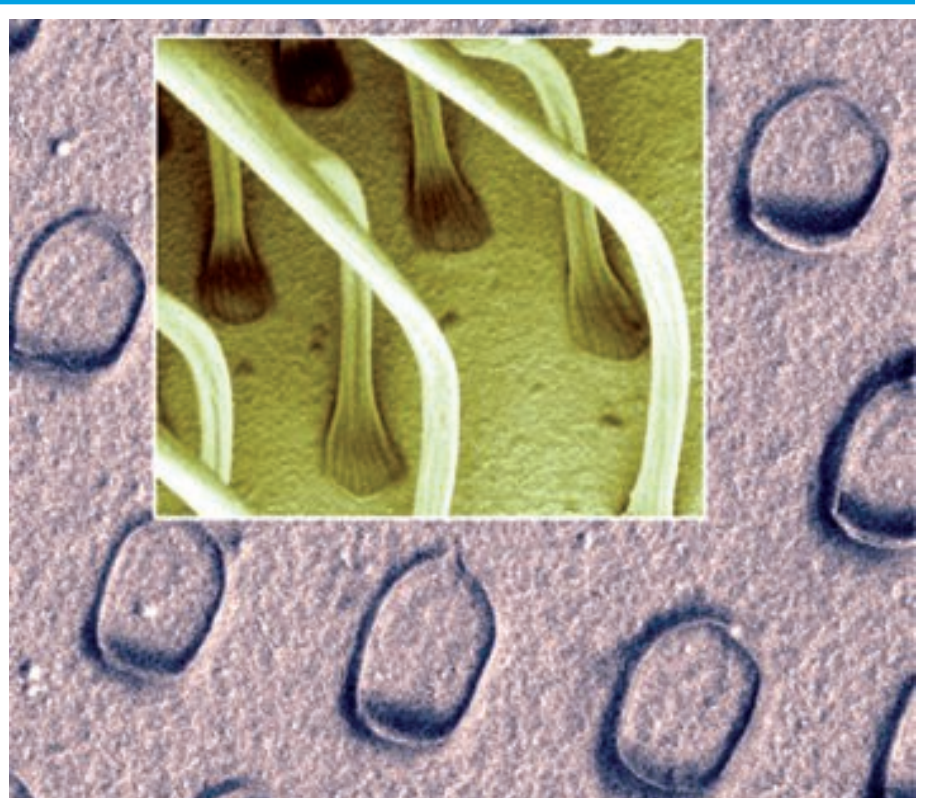

Figure 5. Fluid microdroplets of the fly tenent hairs viewed at the interface between hair tips and metalised polymer substrate, Cryo-SEM. Inset shows tenent hairs in contact)

method can be also used in non-life science applications, such as colloid science, tribology, etc.

\section{Literature}

Gorb, S.N. Attachment devices of insect cuticle. Dordrecht, Boston, London:Kluwer Academic Publishers. 1-305, 2001.

Spurr, A.R. A low-viscosity epoxy resin embedding medium for electron microscopy. J. Ultrastr. Res. 26: 31-43, 1969.

Walker, G., Yule, A.B., and Ratcliffe, J. The adhesive organ of the blowfly, Calliphora vomitoria: a functional approach. J. Zool. Lond. 205: 297-307, 1985.

\section{SII $\odot^{\circ}$ \\ "SCIENTISTSEVERYWHEREAREBEAMING"}

SII NanoTechnology USA Inc.

Silicon Multi-Cathode X-Ray Detector

NO LN2 - Active area $-50 \mathrm{~mm}^{2} *<140 \mathrm{eV}$ FWHM at $5.9 \mathrm{keV} \cdot$ ICR $1.5 \mathrm{Mcps} \cdot$ OCR up to $600 \mathrm{kcps}$

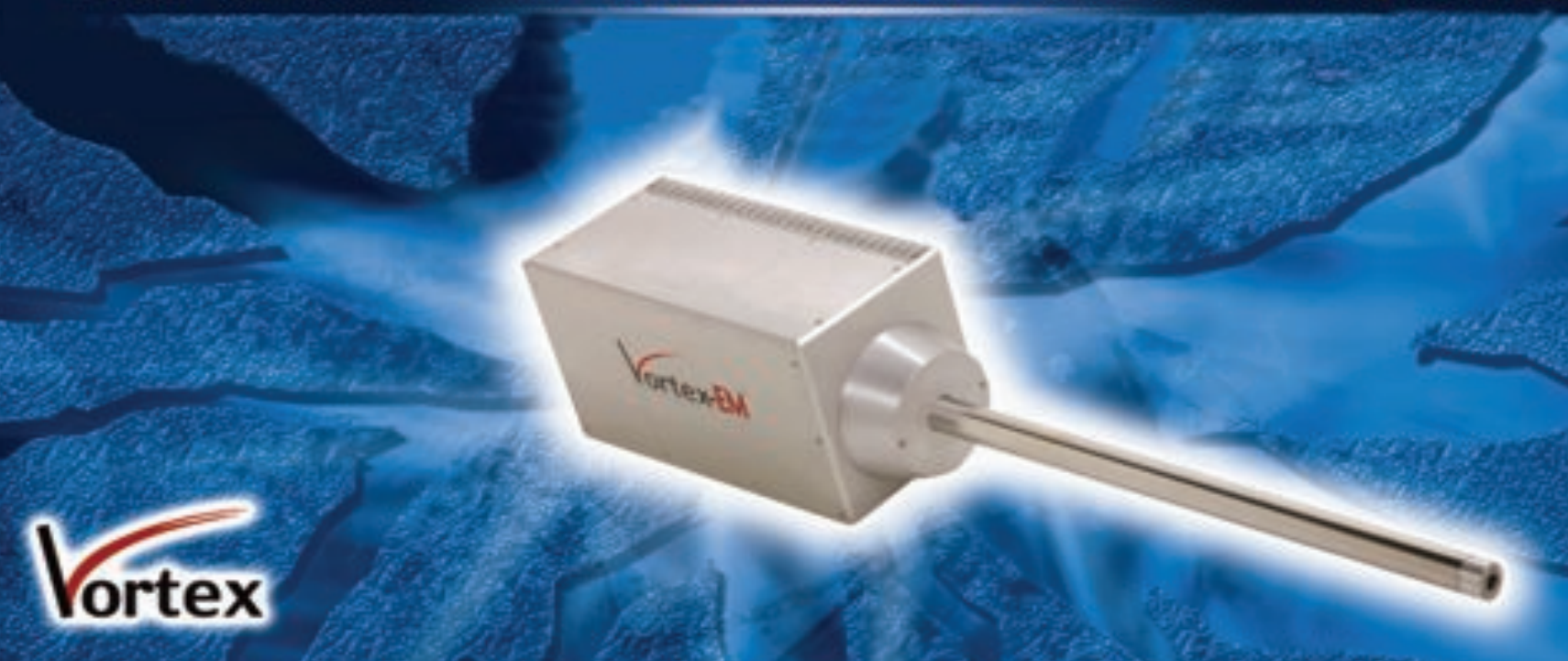

EDS - Fast Mapping - SEM - Microanalysis - Process Control 